\title{
Letter to Editor: "Ultrasonography as an Integrated Tool in Clinical Decision- Making in the Emergency Department"
}

\section{Adela Golea*}

Department of Emergency Medicine, "Iuliu Hatieganu" University of Medicine and Pharmacy, Cluj-Napoca, Romania

According to the American College of Emergency Physicians ultrasound (US) policies, "bedside US exam" is included in clinical protocols in many Emergency Department (ED), especially in USA $[1,2]$. The above-mentioned study introduces the concept of "supporting role of integrated US exam" in diagnosis and clinical decision, with the aim of improving patients' flow. A significant problem in the emergency department is the need for rapid decisions, alongside with low rates of error, therefore ensuring an optimal flow of the patients. In order to ensure a good practice, there must be a constant balance between: time, quality care and evidence based practice. Practitioners usually work with hypothetical-deductive models to develop strategies for good decisions. Hence, the "visual medicine" based on US exam becomes an opportunity to fill the gap which affects this cutting-edge care and offer practitioners a new skill for improving the practice [3]. Further going, US examination is non-radiating. Considering that for many unclear emergency clinical diagnoses, the imagistic gold standard examination is computed tomography, a highly radiating procedure with a significant risk of malignancy over time, US might represent a suitable alternative [4].

\section{Practical Impact}

This study, as well as previous ones, underlines the role of this imagistic investigation on diagnosis of non-differentiated emergencies bearing low clinical concordance [4,5]. Moreover, the results show that early US exam in ED allow rapid decisions regarding the surgical management. The results emphasize the role of receiving US information for patients with co-morbidities and those with alterations detected accidentally, which can influence the short-term prognosis [4-6]. US as a tool used on clinical decision making improves the health care strategy in terms of early diagnosis with reduced errors and urgent therapy, along with associated a rapid flow of patients in ED. We therefore believe that educational programs on integrated ultrasound in clinical assessment of patients designed for emergency medicine residents should be included in the residency program curricula, but also as part of continuing medical education programs $[1,7]$.

\section{Research Impact to Create an Evidence Based Tool for Clinical Decision in ED}

Additional research is need on US exam as an integrated first line imagistic tool for clinical making decisions on all groups of symptoms in order to reduce the time spent and error rates, but also the irradiation of patients in $\mathrm{ED}[2,4,5]$. Literature evidence must be graded to create strong recommendations based on the strength of data from the medical studies. Professional associations of emergency medicine physicians should build a continuous educational program to improve guided clinical assessment of emergencies according to the novel research data.

\section{References}

1. ACEP Board (2016) Ultrasound guidelines: Emergency, point-of-care and critical ultrasound guidelines in medicines.

2. Kennedy H, Taylor A, Luty S, Allen E, Moore Ch (2016) Impact of point-of-care ultrasonography on ED time to disposition for patients with nontraumatic shock. Am J Emerg Med 34: 1022-1030.

3. Medd D (2010) Bedside ultrasound: Part of the physical examination. Am J Med 123: e11.

4. Wytze $L$ (2009) Imaging strategies for detection of urgent conditions in patients with acute abdominal pain: diagnostic accuracy study. BMJ 339: 1-8.

5. Mjolstad OC, Dalen H, Graven T, Kleinau JO, Salvesen O, et al. (2012 Routinely adding ultrasound examinations by pocket-sized ultrasound devices improves inpatient diagnostics in a medical department. Eur J Intern Med 23 185-191.

6. Soultati A, Alexopoulou A, Dourakis SP, Dimopoulou H, Katsaounis P, et al (2010) The burden of incidental findings in clinical practice in a tertiary care center. Eur J Intern Med 21: 123-126.

7. Mandavia D, Aragona J, Chan L, Chan D, Henderson S (2000) Ultrasound training for emergency physicians - A prospective study. Acad Emerg Med 7 : 1008-1014.
*Corresponding author: Adela Golea, Department of Emergency Medicine "Iuliu Hatieganu" University of Medicine and Pharmacy County Emergency University Hospital 3-5 Clinicilor - Street 400006 Cluj-Napoca, Romania, Tel: 00402644318761099; Fax: 0040264599637; E-mail: Adela.Golea@umfcluj.ro

Received April 01, 2016; Accepted April 21, 2017; Published April 28, 2017

Citation: Golea A (2017) Letter to Editor: "Ultrasonography as an Integrated Tool in Clinical Decision-Making in the Emergency Department". Health Care Current Reviews 5: 194. doi: 10.4172/2375-4273.1000194

Copyright: () 2017 Golea A. This is an open-access article distributed under the terms of the Creative Commons Attribution License, which permits unrestricted use, distribution, and reproduction in any medium, provided the original author and source are credited. 